\title{
Palm Temperature Monitoring during Exercise in Patients with Heart Disease
}

\author{
Shunichi Kojima, M.D., Hirotaka Nishijima, M.D., \\ Hisakazu Yasuda, M.D., Masaru Mrnami, M.D., \\ Fuminori Hashimoto, M.D., and Naoya Matsumura, M.D.
}

\section{SUMMARY}

The quantitative use of palm temperature changes during a fixed-load treadmill exercise was evaluated in normal subjects and patients with various degrees of cardiac disability. Treadmill exercise revealed different temperature patterns between subject groups. Normal subjects showed an initial transient decrease to a plateau phase, followed by a prompt return to the control level after cessation of exercise. Cardiac patients with severe disabilities showed a progressive decrease during and even after exercise, and the return to the control level was delayed. Patients with less severe cardiac disabilities showed an intermediate pattern. A significant correlation was observed between the temperature pattern and the plasma catecholamine concentration. The simultaneous measurement of forearm and hand blood flow, and palm temperature during a supine ergometer exercise showed that the temperature change reflected the blood flow changes. In conclusion, palm temperature monitoring during treadmill exercise is a simple and useful method for assessment of the vasoconstrictor response to exercise and, therefore, the pattern of temperature changes indicates indirectly the exercise capacity in heart disease.

\section{Additional Indexing Words:}

Fixed-load exercise Non-exercising limb blood flow Plasma norepinephrine concentration Vasoconstrictor response

$\mathrm{D}$ URING exercise, a major redistribution of organ blood flow occurs as the blood flow to exercising-muscles increases. As a result, blood flow to non-exercising muscles and abdominal organs decreases. An inverse

\footnotetext{
From the Department of Cardiovascular Medicine, Hokkaido University School of Medicine, Sapporo, Japan.

This work was supported by the Research Grant for Cardiovascular Disease (56-C5) from the Ministry of Health and Welfare of Japan.

Address for reprint: Hisakazu Yasuda, M.D., Department of Cardiovascular Medicine, Hokkaido University School of Medicine, Kita-15, Nishi-7, Kita-ku, Sapporo 060, Japan.

Received for publication October 5, 1984.
} 
relationship between the exercising muscle and non-exercising organ blood flow has been observed. ${ }^{11,2)}$ The effect of exercise on skin blood flow is more complex because of the balance between the need for vasodilatation to eliminate heat produced and the tendency towards vasoconstriction due to the increased sympathetic discharge depending on the intensity and duration of exercise. ${ }^{2)}$ However, non-exercising limb blood flow is unique in that it is easily accessible for measurement. Toe or finger temperature measurement at rest has been widely used as an indicator of impaired peripheral circulation in shock. ${ }^{3)}$ In clinical exercise testing, cold sweaty skin has been described as one of the clinical signs indicating impending circulatory failure or a near-maximal point. ${ }^{4}$ However, no attempt has been made so far to utilize this parameter systematically and quantitatively in exercise testing. In this study, we assessed the feasibility of temperature monitoring of the hand during treadmill exercise as an index of impaired peripheral circulation and its utility in evaluating exercise capacity and severity of heart disease.

\section{Materials And Methods}

Materials: The subjects consisted of 13 healthy volunteers (Group A: age $43 \pm 13$, mean $\pm S D$ ), 10 cardiac patients with mild to moderate functional disability of New York Heart Association functional classification (NYHA) I

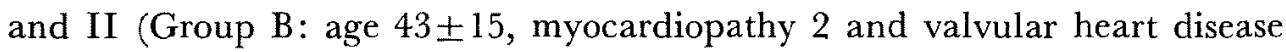
8), and 10 cardiac patients with severe functional disabilities of NYHA III (Group G: age $54 \pm 10$, cardiomyopathy 4 and valvular heart disease 6).

Methods: The temperatures of the palm and forehead were measured by the core-thermistor developed by Fox ${ }^{5)}$ and modified by Togawa ${ }^{6)}$ (CORETEMP, TERUMO). By the heat compensation technique, the type PD3 (diameter $8 \mathrm{~mm}$ ) measures to a depth of $5 \mathrm{~mm}$ from the skin, and the type PD1 (diameter $12 \mathrm{~mm}$ ), $10 \mathrm{~mm}$ from the skin. The accuracy of the thermistor is $\pm 0.1{ }^{\circ} \mathrm{C}$. After attaching the thermistor to the skin (palm and forehead), it takes approximately $10-20 \mathrm{~min}$ before the equilibrium temperature is obtained. Therefore, the subjects rested in the supine position and exercise was begun after the temperature stabilized. The temperature was read from the digital display every minute.

Exercise protocol \#1 (treadmill): All subjects underwent treadmill exercise. The first three stages of the exercise protocol, developed by us for patients with compensated heart failure, were used for both normal and cardiac subjects. The slope and speed of stages $0,1,2$ were respectively as follows: $0 \%, 1.1 \mathrm{mph} ; 10 \%, 1.1 \mathrm{mph} ; 12 \%, 1.6 \mathrm{mph}$ (each stage $3 \mathrm{~min}$ ). The oxygen consumption $(\mathrm{ml} / \mathrm{Kg} / \mathrm{min}$ ) at each stage was as follows: 8.9 , 
12.8, 17.1.7) The total duration of exercise was $9 \mathrm{~min}$ both in normal and cardiac patients. Temperature was measured at the palm, and at the forehead as a reference for the core temperature. EKG was monitored continuously at CM5. Blood pressure was measured with a cuff at 1 min intervals. Blood samples for plasma norepinephrine (NE), epinephrine (E) and renin activity (PRA) were taken from the antecubital vein before exercise (after 25 min of supine bed rest) and also 2 min after the cessation of exercise in the supine position. Plasma NE and $\mathrm{E}$ were determined spectrofluorometrically using the trihydroxyindole method (Shimadzu spectrofluorometer RF-500 LCA). ${ }^{81}$ PRA was determined by radioimmunoassay (Haber).

The temperature of the palm and forehead usually dropped by a variable degree on standing from the supine position. For this reason, the treadmill exercise was started after the stabilization of the temperature.

Analysis of the palm temperature pattern during and after exercise: A schematic representation of the general pattern of the changes in palm temperature is shown in Fig. 1. To aid in analysis of the temperature changes during exercise, the following three parameters were chosen and defined as shown in Fig. 1. Decrease time (DT, endpoint time of decrease) was defined as the elapsed time from the onset of exercise to the point of maximal temperature reduction during or after exercise. Increase time (IT, onset time of increase) was defined as the elapsed time from the onset of exercise to the onset of temperature rise. Recovery time (RT, recovery point time) was defined as the elapsed time from the onset of exercise to the point where the temperature returns to the pre-exercise level after the exercise. When recovery of the temperature to the control level was not observed during the post-exercise observation period, RT was obtained by extrapolation.

Exercise protocol \#2 (supine ergometer): In 5 healthy subjects and 5 cardiac subjects (NYHA I or II), palm temperature and combined forearm and hand blood flow were measured during supine ergometer exercise. A type PD3 thermistor was attached to the left palm (thenar eminence). A mercury strain-gauge plethysmograph was attached to the right forearm, to represent both forearm and hand blood flow; an occlusion cuff at the wrist was not used. Ergometer exercise was done at three successive 3-min stages; $150 \mathrm{kpm}, 300 \mathrm{kpm}$ and $450 \mathrm{kpm}$. Both normal and cardiac subjects exercised for $9 \mathrm{~min}$. During exercise, EKG was monitored at CM5.

Statistical analysis: Paired t-tests were used for paired comparisons. Analysis of variance was used to compare data of more than 2 groups. After that, between-group comparisons were done with a modified t-test (Bonferroni). 


\section{Results}

1. Treadmill exercise

1) Reproducibility and stability of the temperature pattern

The reproducibility of the palm temperature pattern during and after exercise was evaluated in 5 normal and 6 cardiac subjects using the temperature indices as defined above (Fig. 1). The reproducibility was evaluated in two ways: (1) the percent difference calculated as (\#1-\#2)/\#1 measurement $(\%)$, and (2) the absolute difference calculated as \# $1-\# 2$ measurement $(\mathrm{min})$. Variation was expressed as the mean \pm 1 standard deviation (SD). The reproducibility for the percent and absolute differences were: $-27.3 \pm 42.6 \%$ and $-1.1 \pm 2.1 \mathrm{~min}$ for DT, $4.2 \pm 19.1 \%$ and $0.7 \pm 2.2 \mathrm{~min}$ for IT and $-4.5 \pm 24.4 \%$ and $-0.7 \pm 3.8 \mathrm{~min}$ for $\mathrm{RT}$ for the whole group. There was no significant

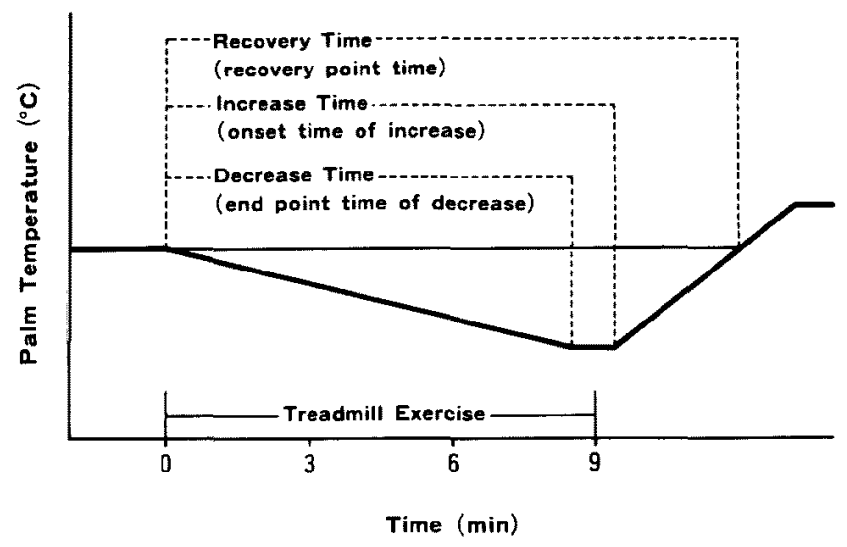

Fig. 1. The definition of decrease time (DT, endpoint time of decrease), increase time (IT, onset time of increase) and recovery time (RT, recovery point time).

Table I. Systolic Blood Pressure and Heart Rate at Rest and Peak Exercise

(Treadmill)

\begin{tabular}{c|cc|cc}
\hline & \multicolumn{2}{|c|}{ SBP $(\mathrm{mmHg})$} & \multicolumn{2}{|c}{ HR } \\
\cline { 2 - 4 } & rest & peak Ex & rest & peak Ex \\
\hline (A) Normal $(\mathrm{n}=13)$ & $113 \pm 14$ & $141 \pm 29^{\mathrm{A}}$ & $67 \pm 13$ & $105 \pm 12^{\mathrm{A}}, \mathrm{B}$ \\
(B) Cardiac, NYHA I, II & $112 \pm 12$ & $128 \pm 18^{\mathrm{A}}$ & $81 \pm 15$ & $143 \pm 28^{\mathrm{A}}, \mathrm{B}$ \\
$(\mathrm{n}=10)$ & $112 \pm 21$ & $140 \pm 29^{\mathrm{A}}$ & $79 \pm 15$ & $137^{\mathrm{A}} \pm 28^{\mathrm{A}}$
\end{tabular}

Abbreviations : $\mathrm{SBP}=$ systolic blood pressure $; \mathrm{HR}=$ heart rate $\mathrm{Ex}=$ exercise $; \mathrm{NYHA}=$ New York Heart Association functional classification.

A : $p<0.01$ vs rest; $B: p<0.05$ vs NYHA III. 
difference in the reproducibility between the normal and cardiac group. The difference in the room temperature and the control palm temperature between $\# 1$ and \#2 measurements was $1.2 \pm 0.9$ and $0.6 \pm 0.6^{\circ} \mathrm{C}$, respectively (not significant). The ranges of the room temperature and resting palm temperature were $23.5-27.8^{\circ} \mathrm{C}$ and $32.2-36.7^{\circ} \mathrm{C}$, respectively. A room tem-

Table II. Control Forehead and Palm Temperature and Absolute Changes after Exercise

(Treadmill)

\begin{tabular}{l|c|c|c|c|c}
\hline & $\begin{array}{c}\text { Forehead } \\
\text { temp } \\
\left.\text { (control, }{ }^{\circ} \mathrm{C}\right)\end{array}$ & $\begin{array}{c}\text { Palm temp } \\
\left.\text { (control, }{ }^{\circ} \mathrm{C}\right)\end{array}$ & $\begin{array}{c}\text { Palm temp } \\
\text { (end of Ex, } \\
\left.{ }^{\circ} \mathrm{C}\right)\end{array}$ & $\begin{array}{c}\text { Palm temp } \\
\text { change } \\
\text { (end of Ex, } \\
\left.\Delta^{\circ} \mathrm{C}\right)\end{array}$ & $\begin{array}{c}\text { Palm temp } \\
\text { change } \\
\text { (maximal } \\
\left.\text { drop, } \Delta^{\circ} \mathrm{C}\right)\end{array}$ \\
\hline (A) $\begin{array}{c}\text { Normal } \\
\text { (n=13) }\end{array}$ & $36.5 \pm 0.3$ & $34.2 \pm 1.0$ & $33.7 \pm 1.3$ & $-0.58 \pm 0.58$ & $-0.75 \pm 0.50$ \\
(B) $\begin{array}{c}\text { Cardiac, } \\
\begin{array}{c}\text { NYHA I, II } \\
\text { (n=10) }\end{array}\end{array}$ & $36.5 \pm 0.4$ & $34.8 \pm 0.9$ & $34.1 \pm 1.1$ & $-0.67 \pm 0.59$ & $-0.92 \pm 0.56$ \\
(C) $\begin{array}{c}\text { Cardiac, } \\
\text { NYHA IJI } \\
\text { (n=10) }\end{array}$ & $36.1 \pm 0.2^{\mathrm{A}}$ & $33.9 \pm 0.9$ & $32.9 \pm 0.9$ & $-0.87 \pm 0.28$ & $-1.31 \pm 0.46$
\end{tabular}

Abbreviations: temp =tempcrature; $\mathrm{Ex}=$ exercise; NYHA=New York Heart Association functional classification; palm temp change (end of $E x$ ) =palm temperature change from the rest value at the end of Ex (in absolute value); palm temp change (max drop)=maximal palm temperature change from control after Ex.

A: $\mathrm{p}<0.05$ vs Normal.

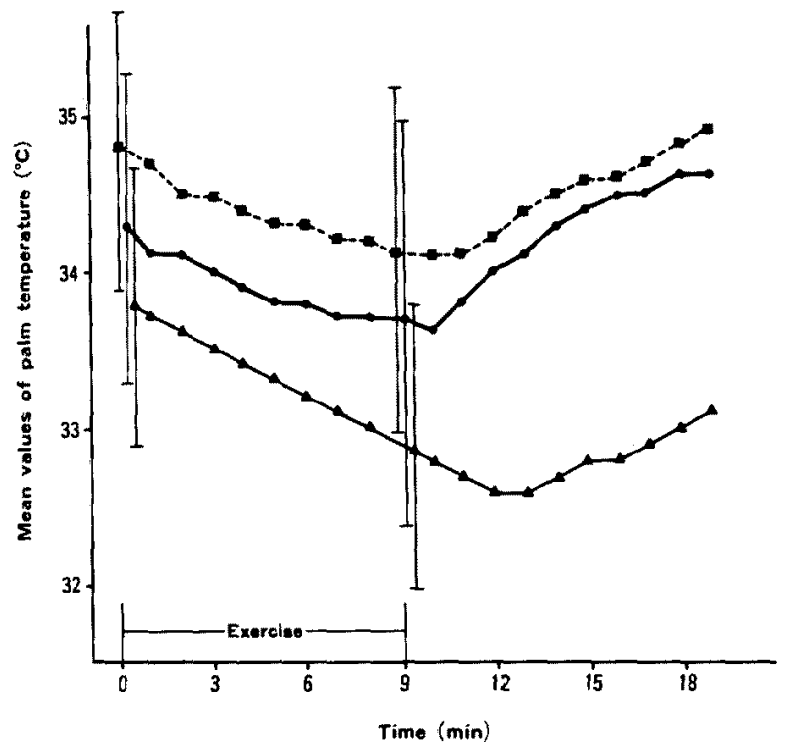

Fig. 2. Mean values of palm temperature before, during and after treadmill exercise in Groups A, B and C. Vertical bars in the figure show mean $\pm S D$ before and at the end of exercise.

B: Cardiac (NYHA I, II);

A: Normal;

- - $\mathbf{a}$ C: Cardiac (NYHA III). 


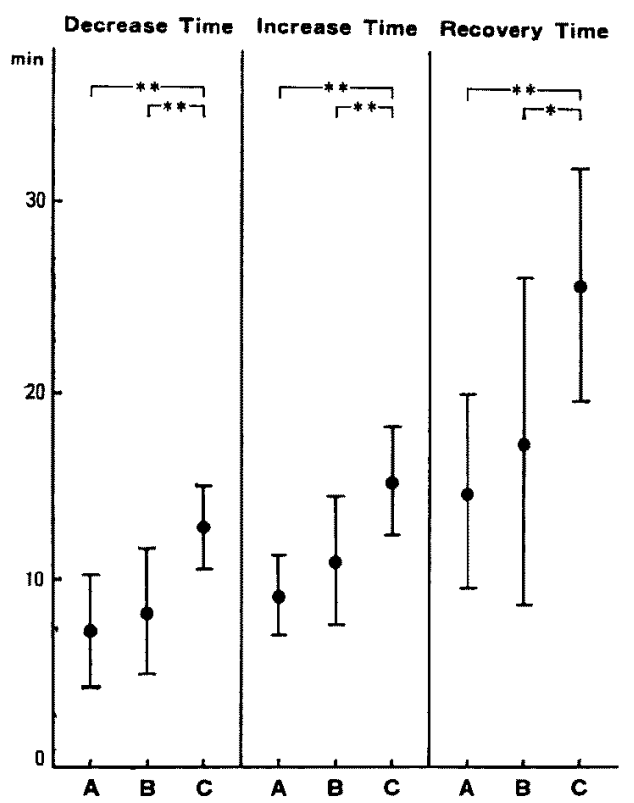

Fig. 3. Values are mean $\pm S D$. Comparison of decrease time, increase time and recovery time among the 3 groups. A: Normal; B: Cardiac (NYHA I, II); C: Cardiac (NYHA III). ${ }^{*} \mathrm{p}<0.05,{ }^{* *} \mathrm{p}<0.01$.

Table III. Plasma Norepinephrine, Epinephrine and Renin Activity before and after Exercise (Treadmill)

\begin{tabular}{|c|c|c|c|c|c|c|c|}
\hline & & \multicolumn{2}{|c|}{$\begin{array}{l}\text { Plasma norepinephrine } \\
(\mathrm{pg} / \mathrm{ml})\end{array}$} & \multicolumn{2}{|c|}{$\begin{array}{l}\text { Plasma epinephrine } \\
(\mathrm{pg} / \mathrm{ml})\end{array}$} & \multicolumn{2}{|c|}{$\begin{array}{l}\text { Plasma renin activity } \\
(\mathrm{ng} / \mathrm{ml} / \mathrm{hr})\end{array}$} \\
\hline & & rest & after $\mathrm{Ex}$ & rest & after Ex & rest & after Ex \\
\hline (A) & $\begin{array}{l}\text { Normal } \\
\quad(n=13)\end{array}$ & $192 \pm 74^{\mathrm{C}}$ & $444 \pm 173^{\mathrm{B}, \mathrm{C}}$ & $42 \pm 29$ & $82 \pm 56^{\mathrm{B}}$ & $1.8 \pm 1.5^{\mathrm{C}}$ & $2.5 \pm 1.5^{\mathrm{B}, \mathrm{C}}$ \\
\hline (B) & $\begin{array}{l}\text { Cardiac, } \\
\text { NYHA I, II } \\
(n=10)\end{array}$ & $240 \pm 109^{c}$ & $591 \pm 310^{\mathrm{B}, \mathrm{C}}$ & $35 \pm 25$ & $47 \pm 35^{A}$ & $3.8 \pm 3.7 \mathrm{c}$ & $8.6 \pm 8.4$ \\
\hline (C) & $\begin{array}{l}\text { Cardiac, } \\
\text { NYHA III } \\
(\mathbf{n}=10)\end{array}$ & $513 \pm 246$ & $1414 \pm 964^{A}$ & $27 \pm 20$ & $50 \pm 25^{\mathrm{A}}$ & $13.9 \pm 11.6$ & $19.7 \pm 16.7^{\mathrm{A}}$ \\
\hline
\end{tabular}

Abbreviations : $\mathrm{Ex}=$ exercise ; NYHA $=$ New York Heart Association functional classification. $A: p<0.05$ vs rest; $B: p<0.01$ vs rest $\quad C: p<0.05$ vs NYHA III.

perature below $23^{\circ} \mathrm{C}$ usually yielded poor reproducibility due to a downward drift of the palm temperature during the control and exercise periods. However, even at higher room temperatures, an erratic downward drift of the control palm temperature was occasionally observed. The experiments at room temperatures $25-27^{\circ} \mathrm{C}$ had the least drift.

2) Palm temperature pattern in the normal and cardiac subjects 
Table IV. Correlations between Humoral Factors and Temperature Parameters

(Treadmill)

\begin{tabular}{|c|c|c|c|c|c|c|}
\hline & $\begin{array}{c}\text { Control } \\
\text { forehead } \\
\text { temp }(\mathrm{n}= \\
\left.30,{ }^{\circ} \mathrm{C}\right)\end{array}$ & $\begin{array}{c}\text { Control } \\
\text { palm temp } \\
\left(n=33,{ }^{\circ} \mathrm{C}\right)\end{array}$ & $\begin{array}{c}\text { Palm temp } \\
\text { change } \\
(\mathrm{n}=33, \text { max } \\
\left.J^{\circ} \mathrm{C}\right)\end{array}$ & 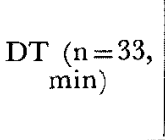 & $\begin{array}{c}\text { IT }(n=33, \\
\min )\end{array}$ & $\underset{\text { min })}{(\mathrm{RT}=33}$ \\
\hline Control NE & $-0.363^{A}$ & $-0.402^{A}$ & 0.276 & $0.499^{B}$ & $0.522^{\mathrm{B}}$ & $0.548^{\mathrm{B}}$ \\
\hline after Ex NE & -0.212 & -0.229 & 0.274 & $0.354 \mathrm{~A}$ & $0.402^{\wedge}$ & $0.487^{\mathrm{B}}$ \\
\hline$\Delta \mathrm{NE}$ & -0.137 & -0.131 & 0.223 & 0.258 & 0.286 & $0.382^{A}$ \\
\hline Control E & $0.355^{A}$ & 0.066 & -0.237 & -0.274 & -0.161 & -0.085 \\
\hline after $\mathbf{E} \times \mathbf{E}$ & $0.387^{\mathrm{A}}$ & -0.012 & -0.261 & $-0.354^{A}$ & -0.269 & -0.286 \\
\hline$\Delta \mathrm{E}$ & 0.328 & -0.082 & -0.210 & -0.329 & -0.294 & -0.300 \\
\hline Control PRA & -0.340 & $-0.426^{\mathrm{A}}$ & 0.285 & $0.455^{\mathrm{B}}$ & $0.471^{\mathrm{B}}$ & $0.477^{\mathrm{B}}$ \\
\hline after Ex PRA & -0.241 & $-0.400^{A}$ & 0.248 & $0.440^{B}$ & $0.499^{\mathrm{B}}$ & $0.519^{\mathrm{B}}$ \\
\hline$\Delta \mathrm{PRA}$ & 0.018 & -0.321 & 0.111 & 0.305 & $0.364^{A}$ & $0.396^{A}$ \\
\hline
\end{tabular}

Abbreviations : temp =temperature $; \mathrm{DT}=$ decrease time ; IT =increase time ; $\mathrm{RT}=$ recovery time ; $\mathrm{NE}=$ norepinephrine $; \mathrm{Ex}=$ exercise $; \mathrm{E}=$ epincphrine $\mathrm{PRA}=$ plasma renin activity $;$ max palm temp change $=$ maximal palm temperature change after exercise from rest value (in absolute value, ${ }^{\circ} \mathrm{C}$ ). $\triangle \mathrm{NE}, \Delta \mathrm{E}, \Delta \mathrm{PRA}=$ the difference: rest-after $\mathrm{Ex}$ (in absolute value).

$A: p<0.05 ; B: p<0.01$.

Blood pressure and heart rate changes during treadmill exercise in healthy and cardiac subjects are summarized in Table I. There were no statistical differences in the mean room temperatures at which the exercise tests were done for the different groups (A: $24.9 \pm 1.1 ; \mathrm{B}: 24.2 \pm 0.9$; and $\mathrm{C}$ : $24.4 \pm 1.5^{\circ} \mathrm{C}$, respectively). Forehead temperature before exercise was significantly lower in Group C. There were no significant differences in the mean palm temperature before exercise between groups (Table II).

The mean palm temperature changes during treadmill exercise in Groups $\mathrm{A}, \mathrm{B}$ and $\mathrm{C}$ are shown in Fig. 2. In Group A, the temperature decrease during exercise tended to level off near the end of exercise, and after exercise a rebound phenomenon was observed. In Group $\mathrm{G}$, the temperature decreased progressively during exercise. This decrease continued well after the exercise and the return to the pre-exercise level was slow. A rebound phenomenon was not observed during the 10 min post-exercise observation period. Group B exhibited an intermediate pattern. Therefore, as shown in Fig. 3, DT, IT and RT were most prolonged in Group C. The absolute decrease in palm temperature was not as useful as the time-sequence changes, because of the large variability (Table II). The forehead temperature changes during or after exercise were usually very small.

3) Changes in plasma catecholamine levels and renin activity during treadmill exercise (Table III)

$\mathrm{NE}$ was significantly higher in Group $\mathrm{C}$ both before and immediately 


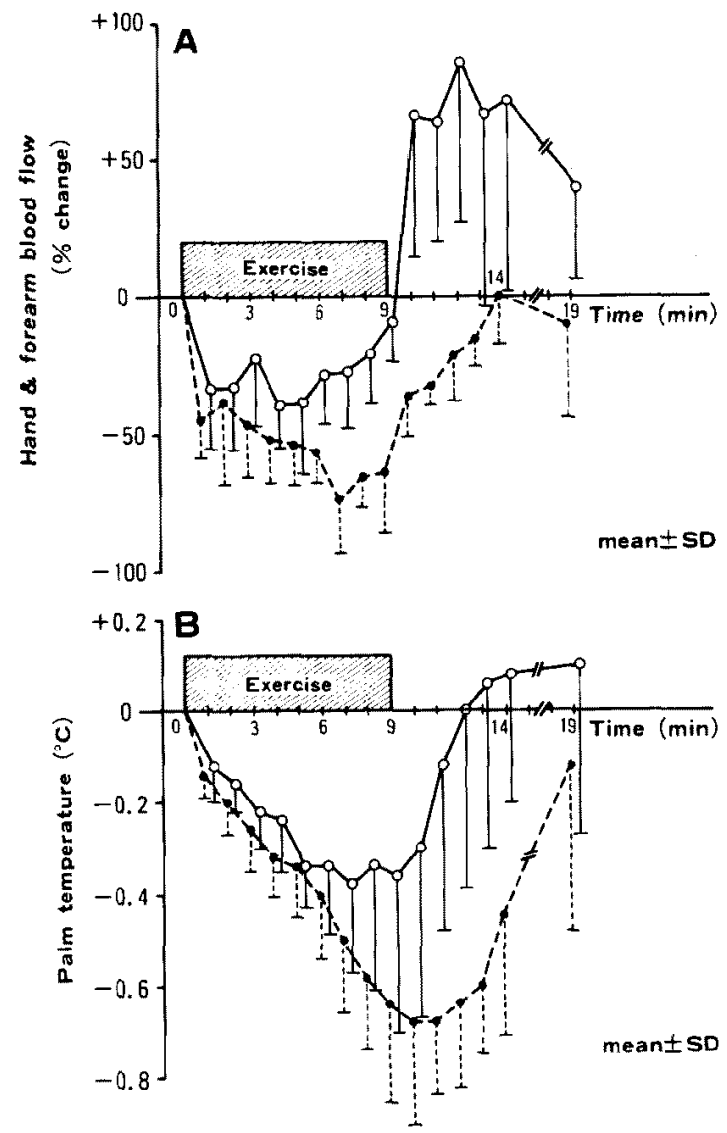

Fig. 4. Values are mean \pm SD. A: \%change of hand and forearm blood flow by plethysmography changes in supine ergometer exercise. B: palm temperature changes by the core temperature thermistor. $\mathrm{O}-\mathrm{O}$ Normal, - Cardiac (NYHA I, II).

after exercise; E showed a similar trend. PRA was also highest in Group C. The absolute changes in NE values in Groups A, B and C induced by exercise were $253 \pm 129,351 \pm 256$ and $901 \pm 947 \mathrm{pg} / \mathrm{ml}$, respectively (A vs C, $\mathrm{p}<0.05)$. PRA also showed a similar trend. The correlation between temperature changes and plasma vasoconstrictor substances is shown in Table IV. The best correlations were obtained between resting NE, PRA and postexercise temperature indices, and also between post-exercise NE, PRA and post-exercise temperature indices, suggesting a close relationship between vasoconstrictor substances and peripheral temperature changes.

2. Ergometer exercise

Fig. 4 shows the changes in hand and forearm blood flow and the changes in the palm temperature during supine ergometer exercise. The temperature changes observed were essentially the same as in treadmill exercise. In 
Table V. Changes in Palm Temperature and Forearm Blood Flow

(Supine Ergometer)

\begin{tabular}{l|cc|cc}
\hline & \multicolumn{2}{|c|}{ Decrease time $(\mathrm{min})$} & \multicolumn{2}{c}{ Changes at decrease time } \\
\cline { 2 - 4 } & \multicolumn{2}{c|}{$\Delta \mathrm{PT}$} & $\% \mathrm{FBF}$ & \multicolumn{2}{c}{$\frac{\Delta \mathrm{PT}\left({ }^{\circ} \mathrm{C}\right)}{\% \mathrm{FBF}(\%)}$} \\
\hline (A) Normal $(\mathrm{n}=5)$ & $6.6 \pm 2.2$ & $4.8 \pm 1.8$ & $0.46 \pm 0.24$ & $-50.0 \pm 14.7$ \\
(B) Cardiac $(\mathrm{n}=5)$ & $10.4 \pm 1.1^{\mathrm{B}}$ & $7.6 \pm 0.5^{\mathrm{A}}, \mathrm{B}$ & $0.74 \pm 0.21$ & $-78.1 \pm 13.1^{\mathrm{B}}$
\end{tabular}

Abbreviations: $\mathrm{PT}=$ palm temperature $; \mathrm{FBF}=$ forearm blood flow.

A : $\quad \mathrm{p}<0.01$ vs $\Delta \mathrm{PT} ; \mathrm{B}: \mathrm{p}<0.05$ vs Normal.

healthy subjects, the initial temperature decrease leveled off towards the end of exercise; after exercise it returned to the pre-exercise level followed by a rebound phenomenon. In cardiac patients the temperature decrease was progressive and continued even after the cessation of exercise. A rebound phenomenon was not observed within $10 \mathrm{~min}$ of the post-exercise period.

The simultaneous blood flow measurements paralleled the temperature data. After an initial decrease, it leveled off and gradually rose to the preexercise level. Immediately after exercise, a marked rebound phenomenon was observed in healthy subjects. In cardiac subjects, though, the blood flow progressively decreased during exercise and the return to the pre-exercise level was delayed after exercise. In general, no prominent rebound phenomenon was seen.

Table V summarizes decrease time (DT) and the palm temperature changes at DT as the blood flow changes in the normal and cardiac subjects. The palm temperature change during and after exercise were closely related to the blood flow changes with some delay.

\section{Discussion}

Exercise causes a re-distribution of blood flow. As the blood flow to exercising muscles increases, the flow to a non-exercising limb and organs such as the liver and kidney decreases. ${ }^{1,2)}$ In cardiac patients, the decrease is more prominent that in normal subjects. ${ }^{9}$ ) However, the general skin blood flow response is more complex than the relatively straightfoward decrease observed in internal organs, because it serves as a heat eliminating system for the heat load generated during exercise.

Since intense exercise invariably induces markedly increased sympathetic discharge which should cause skin vasoconstriction, the skin blood flow response to exercise has two components. The different functional states of the heart may cause a different skin blood flow response to the same work load, depending upon the balance between a sympathetic vasoconstrictor mechanism and a 
heat-mediated vasodilator mechanism. The establishment of an easy method to measure the flow response during exercise testing would be of great aid as an on-line monitor of the functional state of the heart.

In this study, we measured the palm temperature changes during treadmill exercise in 3 different groups of subjects whose clinical cardiac state varied from normal to NYHA III. A pattern which corresponded to the severity of heart disease was observed. The correlation between the level of the plasma vasoconstrictor substances and the palm temperature changes suggested a role of the sympathetic nervous system in determining the blood flow in the non-exercising limb. At rest a decreased finger heat discharge has been demonstrated in heart failure by calorimetry. ${ }^{10)}$

We also demonstrated that during the supine ergometer exercise the non-exercising forearm and hand blood flow tended to level off after an initial decrease and then began to rise even though the exercise intensity progressively increased in normal subjects. This suggests that the need for eliminating heat overrides the vasoconstrictor response, which is still slight at this work load in normal subjects. However, with the same work load in cardiac subjects, the blood flow tended to decrease progressively throughout and even after exercise, suggesting an overriding vasoconstrictor response. This implies that the same work load was probably close to the maximal work load for these cardiac patients. The palm temperature changes closely followed the blood flow changes during and after exercise with some delay, and approximately reflected the blood flow changes. Theoretically, under a given condition, the palm temperature changes should be the net result of the blood flow, the core temperature changes and evaporative heat loss during exercise. However, the observed correlation between the temperature and the blood flow changes indicate the core temperature change was comparatively small (as suggested by the forehead temperature change) and masked by a greater change in the blood flow. Sweating was also limited in this short-term, low-load exercise test. This suggests the use of this simple parameter in place of more complicated blood flow mcasurements. However, Shellock et al ${ }^{11}$ recently reported that during short-term maximal excrcise, the core temperature rose in healthy subjects, but decreased in cardiac patients. This indicates that during maximal exercise, the core temperature changes may grcatly modify the peripheral temperature changes.

Although a transient decrease in non-exercising limb blood flow during leg exercise has been reported, and a steady-state relationship between the skin and core-temperature has been examined by many investigators, ${ }^{12}$ there has not been a previous quantitative study employing peripheral temperature monitoring during exercise testing. The simplicity of palm temperature 
monitoring during exercise as an indicator of vasoconstrictive response or as a non-invasive substitute for plasma catecholamine response is a very attractive concept. However, several problems were encountered in the study. First, although the mean palm temperature response was clearly different between the normal and the cardiac subjects, the reproducibility of the individual response was dependent on the cnvironment. Skin temperature is known to be dependent on the environmental temperature. In this study, our experimental subjects first entered the room and removed clothes from the upper part of the body. These two procedures apparently started a variable downward drifting of the baseline palm temperature, which depended upon the room temperature and the individual's sensitivity to the cold. Although the experiment was done when the room temperature was $23^{\circ} \mathrm{C}-27^{\circ} \mathrm{C}$, the drifting of the palm temperature before exercise was sometimes very prolonged and adequate stabilization was difficult to obtain. Positional changes (e.g., from supine to standing) also induced unpredictable degrees of drift to a lower temperature. The time it took to obtain a stable pre-exercise baseline was the greatest limitation of this method. However, these limitations may be overcome by either conducting the experiment in a temperature-controlled warm environment, by some pre-exercise manuevers or by extending the exercise to the maximal point. The effect of acclimatization on the exercise temperature change in the individual subject was not assessed in this study. However, the individual variation in this respect was not large enough to override the effect of cardiac disability.

In summary, the quantification of peripheral blood flow changes in a non-exercising limb during exercise testing is feasible by the use of a thermistor on the palm. It is a simple and useful method to monitor the vasoconstrictor response to exercise, and therefore indirectly the exercise tolerance of normal subjects and cardiac patients. It may also serve as an approximate, non-invasive substitute for the plasma catecholamine response during exercise.

\section{REFERENCES}

1. Clausen JP: Circulatory adjustments to dynamic exercise and effect of physical training in normal subjects and patients with coronary artery disease. Progr Cardiovasc Dis 18: 459 , 1976

2. Rowell LB: Cardiovascular aspects of human thermoregulation. Circ Res 52: 367, 1983

3. Joly HR, Weil MH: Temperature of the great toe as an indicator of the severity of shock. Circulation 39: 131, 1969

4. Ellestad MH: Stress Testing Principles and Practice. 2nd Ed, FA Davis, Philadelphia, p 32, 1980

5. Fox RH, Solmon AJ, Issac R, Fry AJ, MacDonald IC: A new method for monitoring deep body temperature from the skin surface. Clin Sci 44: 81, 1973

6. Togawa T: A modified internal temperature measurement device. Med Biol Eng 14: 361, 
1976

7. Matsumura N, Nishijima H, Kojima S, Hashimoto F, Minami M, Yasuda H: Determination of anaerobic threshold for assessment of functional state in patients with chronic heart failure. Circulation 68:360, 1983

8. Minami M, Yasuda H, Yamazaki N, Kojima S, Nishijima H, Matsumura N, Togashi H, Koike Y, Saito H: Plasma norepinephrine concentration and plasma dopamine-beta-hydroxylase activity in patients with congestive heart failure. Circulation 67: 1324, 1983

9. Zelis R, Mason DT, Braunwald E: Partition of blood flow to the cutaneous and muscular beds of the forearm at rest and during leg exercise in normal subjects and in patients with heart failure. Circ Res 24: 799, 1969

10. Takano T, Vyden JK, Ogawa T, Rose HB, Marcus HS, Swan HJC: Observations on relationship between digital heat loss and systemic hemodynamics in patients with heart disease. Am Heart J 105: 830, 1983

11. Shellock FG, Rubin SA, Ellrodt AG, Muchlinski A, Brown H, Swan HJG: Usual core temperature decrease in exercising heart-failure patients. J Appl Physiol 54: 544, 1983

12. Johnson JM: Regulation of skin circulation during prolonged exercise. Ann NY Acad Sci 301: 195,1977 